\title{
Ascending-descending aortic bypass surgery in aortic arch coarctation: Four-dimensional magnetic resonance flow analysis
}

Alex Frydrychowicz, MD, ${ }^{a}$ Cristian Schlensak, MD, ${ }^{b}$ Aurélien Stalder, MSc, ${ }^{a}$ Max Russe, ${ }^{a}$ Matthias Siepe, MD, ${ }^{b}$

Friedhelm Beyersdorf, MD, ${ }^{b}$ Mathias Langer, MD, MBA, ${ }^{a}$ Jürgen Hennig, PhD, ${ }^{a}$ and Michael Markl, PhD, ${ }^{a}$ Freiburg, Germany

Supplemental material is available online.

$\int$ n light of the current discussion about the effect of blood flow on arterial wall remodeling, ${ }^{1-3}$ the investigation of vascular hemodynamics within the aorta is of great interest. It has the potential to greatly enhance the understanding of the pathogenesis and progression of vascular diseases and to aid in the decision of

From the Departments of Diagnostic Radiology and Medical Physics ${ }^{\mathrm{a}}$ and Cardiovascular Surgery, ${ }^{\mathrm{b}}$ University Hospital Freiburg, Freiburg, Germany.

Received for publication Aug 9, 2006; accepted for publication Aug 25, 2006.

Address for reprints: Alex P. Frydrychowicz, MD, University Hospital Freiburg, Department of Diagnostic Radiology, Hugstetter Str. 55, 79106 Freiburg, Germany (E-mail: alex.frydrychowicz@uniklinik-freiburg.de).

J Thorac Cardiovasc Surg 2007;133:260-2

$0022-5223 / \$ 32.00$

Copyright $\odot 2007$ by The American Association for Thoracic Surgery doi:10.1016/j.jtcvs.2006.08.061 whether treatment is warranted. Noninvasive magnetic resonance imaging (MRI), with its intrinsic sensitivity to blood flow, offers the unique possibility to simultaneously acquire morphology and spatially coregistered hemodynamic information noninvasively. ${ }^{4}$

Electrocardiography-synchronized and respiration-controlled 3-dimensional magnetic resonance (MR) velocity mapping (flowsensitive 4-dimensional MRI), a state-of-the-art MRI technique, was used to acquire time-resolved, 3-dimensional, morphologic, and 3-directional blood flow data. Visualization was performed with a commercially available software tool initially developed for engineering applications, which allowed for several 3-dimensional visualization modes for a detailed visual inspection of local hemodynamic features associated with complex vascular geometries (Appendix E1). ${ }^{5}$ A homemade software tool was used to quantify blood flow information at multiple locations along the vascular tree.

Based on an exemplary patient measurement, the possibilities of time-resolved 3-dimensional flow visualization and MR-based quantitative flow analysis are demonstrated, which might influence therapeutic strategies in individual patients in addition to standard clinical diagnostic testing.

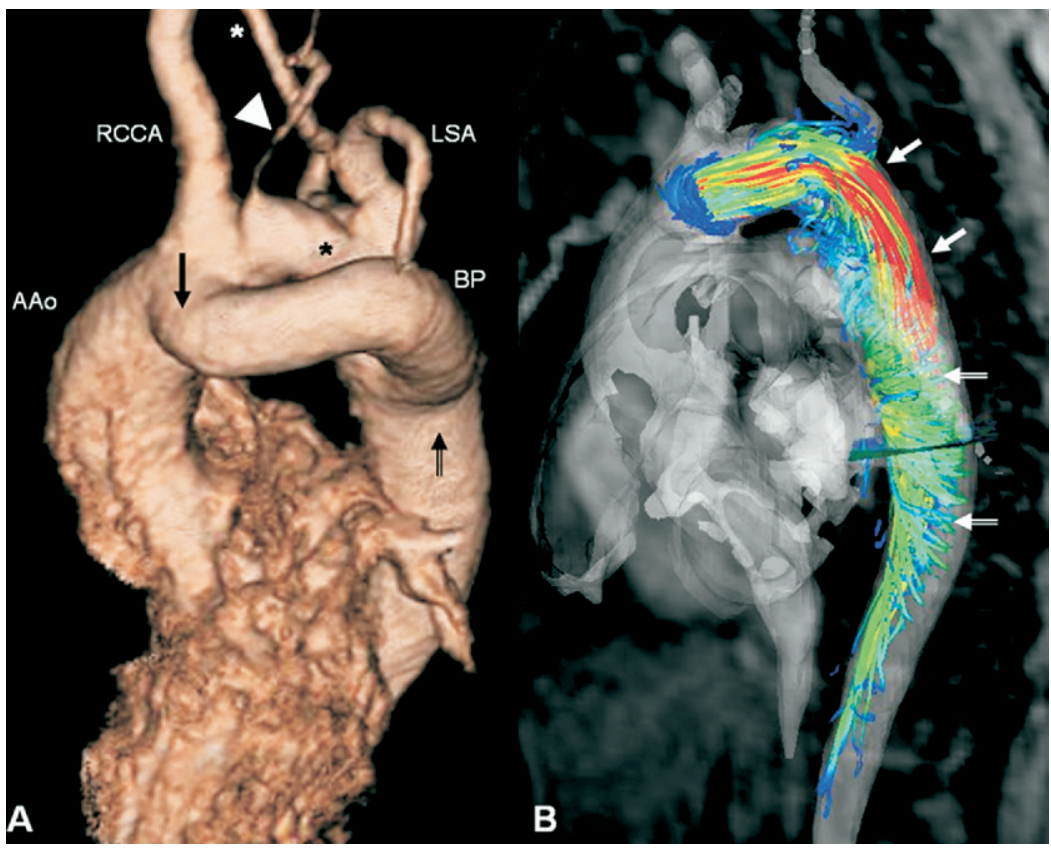
asterisk, site of coarctation.
Figure 1. A, Volume-rendered images from 3-dimensional contrast-enhanced magnetic resonance angiography at $3 \mathrm{~T}$. The bypass from ascending to descending aorta (closed to open black arrow) can be clearly appreciated. The coarctation is situated at the height of an atypical origin of a lusoric artery to the right arm (white asterisk) and the left subclavian artery. Note the large diameter of the right common carotid artery and the small left internal carotid artery as the second supra-aortic branch (white arrowhead). The left external carotid artery originates distal to the coarctation and is not displayed here. B, Three-dimensional streamline visualization of vascular hemodynamics in the aortic bypass graft and the proximal descending aorta at peak systole. Color coding corresponds to absolute flow velocities. Note the accelerated flow in the bypass. Also, considerable helical flow as a result of surgically altered vascular geometry can be appreciated in the descending aorta. See also Figure E1 for a detailed analysis of blood flow patterns by means of 3-dimensional particle trace visualization. AAo, Ascending aorta; $R C C A$, right 


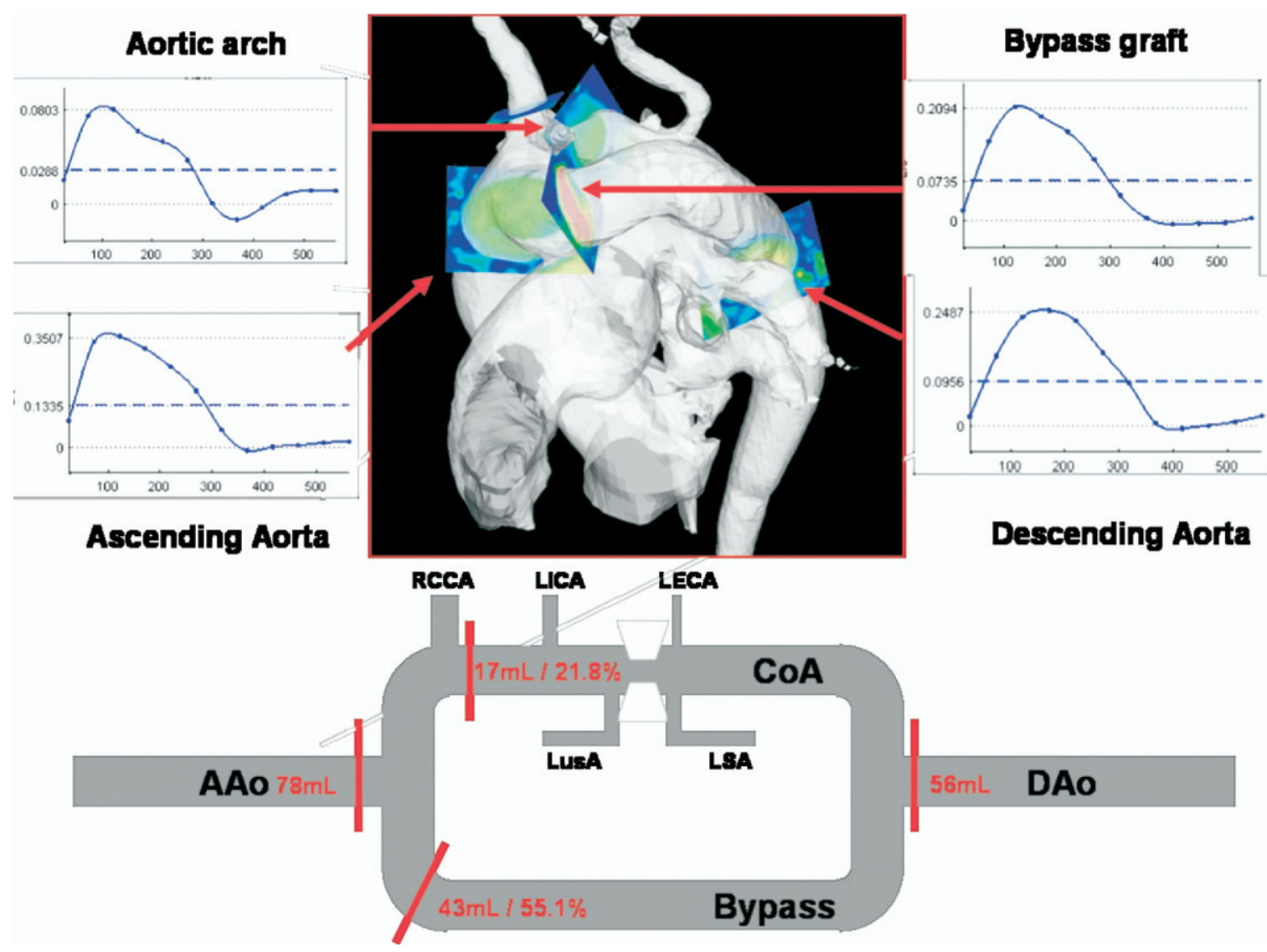

Figure 2. Middle, surface-rendered image from flow-sensitive 4-dimensional magnetic resonance imaging with cut planes placed orthogonal to the general flow direction. Left and right, Charts displaying blood flow over time in liters per millisecond in the cardiac cycle for 12 acquired time frames. Below, Flow scheme displaying blood flow per cardiac cycle distributed within the vessels of interest. Cut planes used for flow quantification and absolute results, as well as percentage of flow through bypass and arch, are schematically indicated by red bars and red numbers, respectively. $A A O$, Ascending aorta; $C O A$, coarctation; $D A o$, descending aorta; $R C C A$, right common carotid artery; $L I C A$, left internal carotid artery; $L E C A$, left external carotid artery; $L S A$, left subclavian artery; $L$ Ls $A$, lusoric artery.

\section{Anatomic Findings and Clinical Summary}

In this symptomatic patient MR angiography depicted a moderate coarctation of the aortic arch (Figure 1). However, in addition to the stenosis, unusual origins of the supra-aortic branches were diagnosed. A large right common carotid artery was directly followed by the left internal carotid artery. At the height of the coarctation, both the left and right subclavian arteries originated. The right subclavian artery crossed behind the esophagus to the right side as a lusoric artery (Figure 1, white asterisk). Directly distal to the stenosis, the left external carotid artery (Figure 2) branched from the aorta.

The patient was offered surgical repair of the aorta because the atypical aortic branches are associated with an increased risk for complications during endovascular intervention. By using an intercostal approach, a 2-cm Dacron bypass graft was placed between the ascending and descending aortas. Postoperatively, the patient complained about dizziness and became unconscious once.
However, cranial computed tomography and color-coded Doppler ultrasonography of the external and internal carotid artery remained unchanged.

\section{D MR Flow Analysis}

Four-dimensional MR velocity mapping was performed for an additional overview over the flow alterations and hemodynamic status after the surgical procedure. Figure $1, B$, resembles particle traces at late systole. The highly accelerated flow within the bypass graft can be clearly appreciated. Additionally, overt vertical and considerable helical blood flow in the descending aorta distal to the bypass anastomosis were observed. Severe blood flow alterations, even further downstream to the site of the bypass, points toward the effect of the performed surgical procedure and the long-term alteration of vessel wall parameters because the helical flow is associated with altered wall shear rates. Also, the efficiency of 
vascular filling expressed by the energy loss caused by enhanced transverse kinetic energy is jeopardized and might lead to a changed distribution of blood flow further downstream. (These findings can be appreciated in more detail by viewing the timeresolved particle traces shown in Figure E1.)

For a more quantitative analysis, multiple extracted cut planes were evaluated manually by means of individual segmentation of the vascular lumen at all time frames within the cardiac cycle. Results are given in Figure 2 for four 2-dimensional cut planes in the ascending aorta before the bypass, in the descending aorta distal to the bypass, and for both the aortic arch and the proximal bypass (see also schematic analysis, Figure 2, below). The amount of blood flow was readily computed and is displayed as corresponding flow curves with unsuspicious pulse-wave diagrams. Remarkably, about $55 \%$ of ascending aortic blood flow volume was directed through the bypass, whereas only $22 \%$ was delivered to the aortic arch and thereby to the supra-aortic branches. This "aortic steal" effect led to the decision to introduce $\alpha_{1}$-mimetics. Using this medication, the patient had a relief of neurological symptoms in her daily life.

\section{Discussion}

Despite the limitations, such that MRI is not as widely available as ultrasonography and not ubiquitously available, the technique offers a unique possibility for comprehensive assessment of 3- dimensional vascular hemodynamics, especially in complex vascular geometries. More notably, the added information content not assessable with other imaging modalities has the potential to influence therapeutic decision making, as in the case presented here. However, further serial assessments and patient studies are necessary to evaluate the potential to influence therapeutic decision making and planning of operative procedures in vascular disease.

\section{References}

1. Richter Y, Edelman ER. Cardiology is flow. Circulation. 2006;113 2679-82.

2. Cheng C, Tempel D, van Haperen R, et al. Atherosclerotic lesion size and vulnerability are determined by patterns of fluid shear stress. Circulation. 2006;113:2744-53.

3. Wentzel JJ, Corti R, Fayad ZA, et al. Does shear stress modulate both plaque progression and regression in the thoracic aorta? Human study using serial magnetic resonance imaging. J Am Coll Cardiol. 2005;45: 846-54.

4. Pelc LR, Pelc NJ, Rayhill SC, et al. Arterial and venous blood flow: noninvasive quantitation with MR imaging. Radiology. 1992;185:80912 .

5. Frydrychowicz A, Weigang E, Harloff A, et al. Images in cardiovascular medicine. Time-resolved 3-dimensional magnetic resonance velocity mapping at $3 \mathrm{~T}$ reveals drastic changes in flow patterns in a partially thrombosed aortic arch. Circulation. 2006;113:e460-1. 


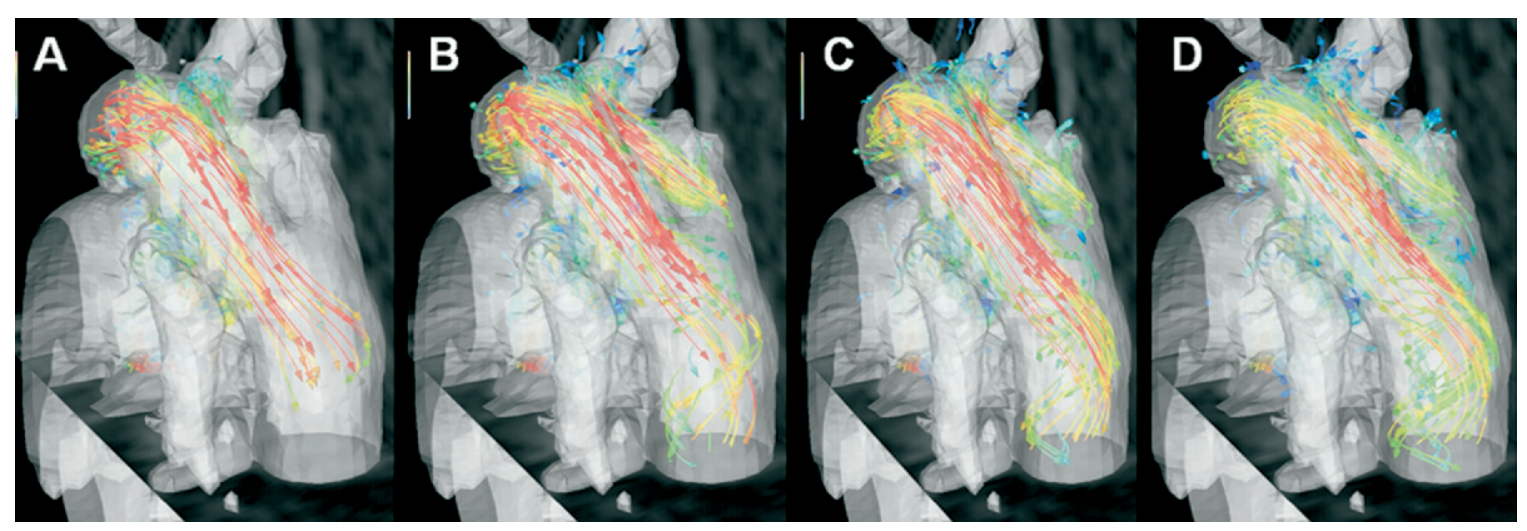

Figure E1. Time-resolved 3-dimensional particle trace visualization shows the blood flow characteristics through the bypass graft at 4 points during systole. Note the induction of helical flow in the descending aorta and the high flow velocities directed through the bypass in comparison with the stenosed aortic arch.

\section{Appendix E1. Additional Information on methodology}

The investigation was performed on a 3T-MR-scanner (TRIO; Siemens Medical Solutions, Erlangen, Germany) using a previously described and modified radiofrequency-pulse spoiled gradient echocardiographic sequence with interleaved 3-directional velocity encoding. Imaging parameters were as follows: velocity encoding, $150 \mathrm{~cm} / \mathrm{s}$; spatial resolution, $2.1 \times 3.2 \times 3.5 \mathrm{~mm}^{3}$; sagittal oblique field of view, $400 \times 300 \mathrm{~mm}^{2}$; flip angle, $15^{\circ}$; echo time, $3.5 \mathrm{~ms}$; repetition time, $6.1 \mathrm{~ms}$; bandwidth, $480 \mathrm{~Hz}$ per pixel. Respiratory-controlled imaging was performed based on adaptive navigator gating, allowing for free breathing. After the MR scan, the acquired flow-sensitive 4-dimensional MR data underwent fully automated noise filtering and eddy current correction.

For visualization, a commercially available software package (EnSight; CEI, Apex, NC) was used. Visualization options included 3-dimensional streamlines, time-resolved 3-dimensional particle traces, and 2-dimensional time-resolved vector fields. With these visualization options, a temporal and spatial description of flow characteristics in 3 dimensions was performed. 